Reprod. Nutr. Dévelop., 1983, 23 (1), 13-24.

\title{
Approche méthodologique de la répartition nycthémérale des prises d'aliment chez le lapin domestique en croissance
}

\author{
E. JOLIVET, Y. REYNE $\left({ }^{*}\right)$, J. TEYSSIER $\left({ }^{*}\right)$
}

avec la collaboration technique de G. VAN BORREN et Suzanne PAOLANTONACCI $\left({ }^{*}\right)$

Laboratoire de Biométrie, I.N.R.A., 78350 Jouy-en-Josas, France.

(*) Station de Physiologie animale, I.N.R.A.-E.N.S.A. 34060 Montpellier Cedex, France.

Summary. A methodological approach to the circadian pattern of food intake in the growing domestic rabbit.

We graphically recorded the feeding pattern of 9 New Zealand male rabbits during weeks $6,9,12,15$ and 18 of age. Feeding was considered as a periodic series of discrete events. We fitted a periodic Poisson process to each particular series. In order to determine if the model was a good representation of the time series, we compared the estimated density curves with non-parametric estimations of density. Approximation of the circadian pattern using the model was better from week 15 on. Before this age, 24-hour feeding activity was generally characterized by two peaks.

The parameters of the model - average number of food intakes over a 24-hour period, time of highest feeding activity and scatter index - were easy to interpret. Age variations in circadian feeding pattern were mainly characterized by shortening of scatter and shifting of peak feeding activity from the beginning to the middle of the dark period between weeks 6 and 18 .

\section{Introduction.}

Chez le Lapin, la caractéristique la plus évidente du comportement alimentaire est la présence d'un rythme d'ingestion sur la période de $24 \mathrm{~h}$ qui a été décrit par de nombreux auteurs (Horton et al., 1974 ; Prud'hon et al., 1975a ; Sanderson et Vanderweele, 1975 ; Hörnicke et al., 1976).

Dans la plupart des situations étudiées, il semble que la répartition nycthémérale des consommations soit pratiquement identique à celle du nombre de prises d'aliment (Prud'hon et al., 1975a et b ; Prud'hon, 1976).

C'est pourquoi l'étude dans ce premier article de l'évolution du rythme d'ingestion chez des lapins domestiques en croissance par l'analyse des seuls instants de prise nous a paru intéressante. Dans ce but nous avons développé une approche méthodologique relevant de la statistique des processus ponctuels et 
donnant une description de la séquence des instants de prise d'aliment par des paramètres aisément interprétables.

\section{Matériel et méthodes.}

\section{Conditions expérimentales.}

Neuf lapins mâles de race Néo-Zélandaise ont été placés dans des cages individuelles équipées d'un dispositif d'enregistrement graphique des consommations. Ce dispositif a été décrit en détail par Prud'hon et al. (1972) ; il permet de mesurer avec une erreur inférieur à 2 min les intervalles de temps séparant la fin de deux prises d'aliment successives.

Le terme de "prise d'aliment » est employé dans cet article au sens où il est utilisé par Prud'hon et al. (1975a). Une «prise d'aliment " correspond à tout acte de consommation détectable par le système d'enregistrement utilisé et séparé du précédent par une durée minimum de $45 \mathrm{sec}$. "L'instant de prise " est le moment où la consommation est enregistrée, soit la fin de la prise d'aliment.

Les animaux ont été sevrés à 4 semaines, acclimatés aux conditions expérimentales durant une semaine puis enregistrés au cours des $6^{\mathrm{e}}, 9^{\mathrm{e}}, 12^{\mathrm{e}}, 15^{\mathrm{e}}$ et $18^{\mathrm{e}}$ semaines. Pour chaque âge, on a ainsi une série d'instants de prise correspondant à 7 jours consécutifs d'enregistrement.

Les cages sont placées dans deux pièces parfaitement isolées de la lumière extérieure et climatisées, la température étant maintenue constante à $20^{\circ} \pm 1^{\circ} \mathrm{C}$. L'éclairement d'une intensité de 1000 lux au niveau des cages est assuré par des tubes fluorescents de type lumière du jour. Les animaux ont été éclairés de $7 \mathrm{~h}$ à $21 \mathrm{~h}$ pendant toute la durée de l'expérience, soit un rapport lumière-obscurité de $14 / 10$. Ils recevaient à volonté de l'eau et un aliment granulé du commerce dosant $15 \%$ de protéines brutes et $14 \%$ de cellulose.

\section{Hypothèses de travail.}

Les données de comportement alimentaire se présentent comme une succession plus ou moins régulière de prises d'aliment. Dans le cas du rat, il existe une certaine tendance à l'agrégation des prises qui suggère la présence de repas et a conduit les chercheurs à s'interroger sur l'existence de mécanismes responsables du déclenchement du repas ou de son arrêt. Le calcul de corrélations pour déterminer si la dimension des repas est liée à la durée des intervalles qui les précèdent ou à la durée des intervalles qui les suivent a reçu une grande attention (Le Magnen et Tallon, 1966 ; Thomas et Mayer, 1968 ; De Castro, 1975, 1978 ; Le Magnen et Devos, 1980), de même que le calcul de différents paramètres sur la période diurne et la période nocturne (quantités ingérées, nombre de repas, taille des repas).

Cette méthode d'analyse a fait l'objet de nombreuses controverses (Panksepp, 1978) et oublie que le phénomène étudié se présente comme une série d'événements dont on doit prendre en compte la structure temporelle. Pour per- 
mettre une meilleure approche de cet aspect, nous avons analysé l'évolution avec l'âge du rythme d'ingestion chez le Lapin en supposant que les instants d'apparition de chaque prise individuelle d'aliment sont distribués comme les points d'un processus de Poisson périodique de densité $h(t)$.

A priori rien ne s'oppose à cette hypothèse. En effet, si la représentation graphique des données obtenues pour les prises d'aliment d'un lapin à différents âges met bien en évidence la périodicité, elle ne permet pas de déceler une tendance à l'agrégation des prises individuelles (fig. 1).


semaine

maine
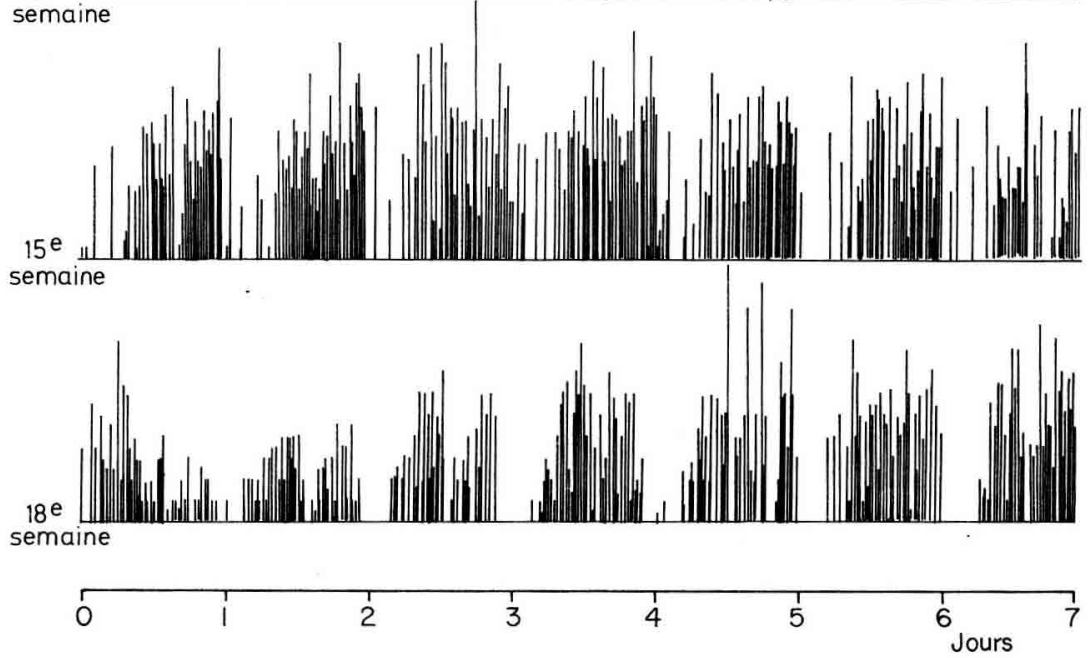

FIG. 1. - Représentation graphique de l'activité alimentaire.

Exemple pour un lapin domestique enregistré au cours de 7 jours consécutifs à différents âges. 
$D^{\prime}$ ailleurs la forme de l'histogramme de fréquence des durées de pause entre prises d'aliment diffère nettement de celle observée chez le Rat, essentiellement par une fréquence beaucoup plus faible des intervalles de courtes durées (Prud'hon et al., 1972 ; Prud'hon et al., 1975a ; Sanderson et Vanderweele, 1975).

\section{Méthodes utilisées.}

L'utilisation du périodogramme des instants et l'estimation non paramétrique de la densité $h(t)$ (cf. annexe) permettent de vérifier l'existence d'une périodicité et fournissent des renseignements sur la répartition des prises d'aliment au cours du nycthémère.

Le processus de Poisson périodique et la méthode d'estimation des paramètres ont déjà été décrits par Jolivet (1980). Rappelons simplement que dans la pratique courante, la densité choisie pour le processus de Poisson périodique est :

avec $\omega=\frac{2 \pi}{\mathrm{T}}, \mathrm{T}$ étant la période du phénomène.

$$
h(t)=\exp \left(a+b \cos \omega\left(t-t_{\varphi}\right)\right] / I_{0}(k)
$$

$h(t)$ peut également se mettre sous la forme :

$$
h(t)=\lambda \exp \left[k \cos \omega\left(t-t_{\varphi}\right)\right] / I_{0}(k)
$$

où $\mathrm{I}_{0}(\mathrm{k})=\frac{1}{\mathrm{~T}} \int_{0}^{\mathrm{T}} \exp [\mathrm{k} \cos \omega(\mathrm{t}-\mathrm{t} \varphi)] \mathrm{dt}$

Dans ce cas les paramètres $\lambda$, $\mathrm{k}$ et $\mathrm{t}_{\varphi}$ ont une signification bien précise : $-\lambda$ représente le nombre moyen de prises d'aliment par unité de temps calculé sur une période ;

- th est l'instant où $h(t)$ est maximum c'est-à-dire l'instant où la fréquence des prises est la plus élevée (activité alimentaire maximum) ;

- $k$ est un indice d'étalement de l'activité alimentaire : plus $k$ est grand plus cette activité est concentrée, c'est-à-dire répartie sur une petite fraction du nycthémère.

En supposant que les estimateurs obtenus pour chacun des paramètres sont des réalisations indépendantes d'une variable aléatoire gaussienne on peut utiliser l'analyse de variance pour étudier l'effet âge et l'effet lapin sur les estimateurs $\hat{\lambda}$, $\hat{k}$, et $\hat{t}_{\varphi}$. Le modèle général retenu est un modèle croisé à deux effets fixés :

$$
P_{i j}=\mu+\alpha_{i}+\beta_{j}+e_{i j}
$$

où $\quad \mathrm{P}_{\mathrm{ij}} \quad$ est la valeur du paramètre étudié pour la jième semaine et le lapin $\mathrm{j}$

$\mu \quad$ est la moyenne générale

$\alpha_{i}$ est l'effet âge i (i $\left.=1, \ldots, 5\right)$

$\beta_{\mathrm{j}} \quad$ est l'effet lapin $\mathrm{j}(\mathrm{j}=1, \ldots, 9)$

$\mathrm{e}_{\mathrm{ij}} \quad$ est l'effet résiduel, $\mathrm{e}_{\mathrm{ij}} \sim \mathcal{N}\left(0, \sigma^{2}\right)$ 


\section{Résultats.}

\section{Périodogramme.}

L'analyse à l'aide du périodogramme de chacune des séries d'instants de prise obtenues aux différents âges confirme d'une façon très nette la périodicité de $24 \mathrm{~h}$, sauf dans un cas, pour un lapin âgé de 6 semaines. La figure 2 présente un exemple typique de périodogramme avec un pic très important à la fréquence correspondant à la période de $24 \mathrm{~h}$.

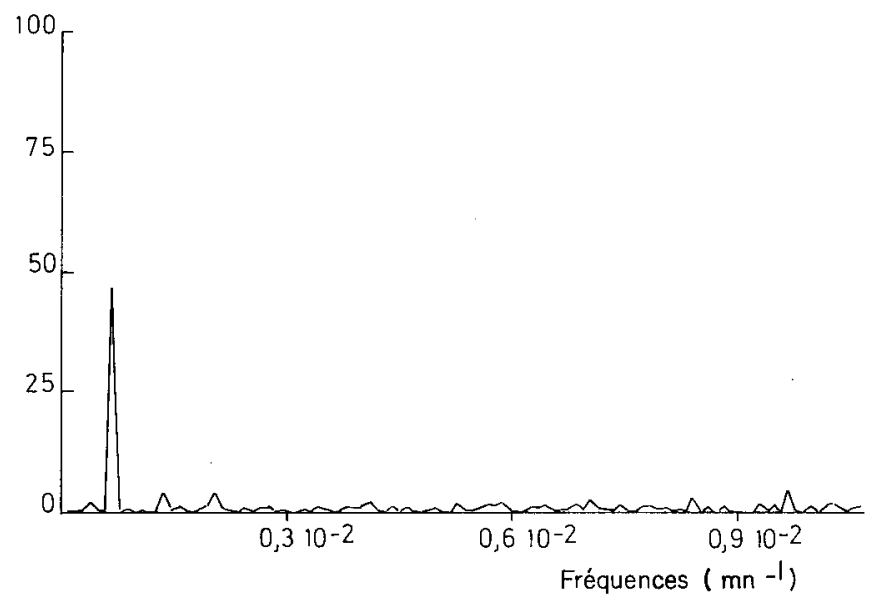

FIG. 2. - Périodogramme des instants.

Exemple pour un lapin enregistré au cours de sa $15^{\mathrm{e}}$ semaine.

\section{Estimations non paramétriques de la densité.}

Les estimations non paramétriques de la densité permettent de visualiser l'évolution de la répartition nycthémérale des instants de prise d'aliment entre la $6^{\mathrm{e}}$ et la $18^{\mathrm{e}}$ semaine. La figure 3 présente à titre $d^{\prime}$ exemple les estimations de la densité obtenues à différents âges pour un des lapins enregistrés. On note chez tous les animaux et à tous les âges, l'existence d'une période bien marquée de moindre activité durant la phase d'éclairement. Ce minimum d'activité tend à se déplacer avec l'âge du début vers le milieu de la phase d'éclairement.

Pour sept des animaux enregistrés, 2 pics d'activité sont présents à l'âge de 6 semaines. Ces deux pics existent chez tous les animaux à 9 semaines. Le premier pic se situe toujours dans les heures qui précèdent l'extinction de la lumière. II est suivi par une deuxième phase de diminution d'activité beaucoup moins marquée que celle observée en début de période d'éclairement, sauf dans le cas d'un animal où cette deuxième phase de moindre activité est également très prononcée. Le caractère bimodal de la répartition des prises d'aliment s'estompe 




FIG. 3. - Estimations non paramétriques de la densité obtenues à différents âges. Exemple d'un lapin.
$6^{\text {e }}$ semaine :
; ge semaine ; $12^{e}$ semaine :
$15^{\mathrm{e}}$ semaine :
$18^{\mathrm{e}}$ semaine : $\ldots \ldots$.... La zone ombrée correspond à lá période d'obscurité

peu à peu avec l'âge des animaux et à 18 semaines, l'activité présente un seul pic dont le sommet se situe généralement au milieu de la phase obscure.

\section{Etude à l'aide du modèle " usuel » de processus de Poisson périodique}

3.1. Estimation des paramètres. - Les résultats obtenus pour l'estimation des paramètres sont donnés dans le tableau 1.

A la vue de ces valeurs, on est tenté de dire que la période d'activité alimentaire maximale se déplace du début vers le milieu de la nuit et que l'étalement de cette activité est de plus en plus réduit lorsque le lapin vieillit. Par contre, l'évolution des valeurs observées pour le nombre journalier de prises d'aliment est moins évidente. L'analyse de variance va nous permettre de préciser ces hypothèses.

3.2. Influence des facteurs "âge " et " animal " sur les paramètres. - Au niveau de $5 \mathrm{p}$. 100, les tests usuels sur le modèle a priori d'analyse de variance mettent en évidence un effet lapin significatif pour $\hat{\lambda}$ et $\hat{k}$ et un effet âge significatif pour $\hat{k}$ et $\hat{t}_{\varphi}$. Pour ces deux derniers paramètres, la variation des $\alpha_{i}$ en fonction de l'âge permet d'affiner le modèle en introduisant l'âge $t_{i}$. Dans le cas de l'expérience considérée nous avons finalement retenu pour décrire les 3 estimateurs, les modèles suivants :

$$
\begin{aligned}
\text { - } \hat{\lambda}_{i j}= & \mu+\beta_{j}+e_{i j} \quad e_{i j} \sim \mathcal{N}\left(0, \sigma^{2}\right) \\
& \text { avec } \hat{\mu}=38,9 \\
\hat{\sigma} & =7,3 \\
& \text { où } \hat{\mu} \text { et } \hat{\sigma} \text { sont respectivement les estimateurs de } \mu \text { et } \sigma
\end{aligned}
$$


TABLEAU 1

Valeurs des estimateurs des paramètres obtenues à l'aide du modèle de processus de Poisson périodique pour 9 lapins domestiques à différents âges.

\begin{tabular}{|c|c|c|c|c|}
\hline $\begin{array}{c}\mathrm{N}^{\circ} \\
\text { lapin }\end{array}$ & $\begin{array}{c}N^{\circ} \\
\text { semaine }\end{array}$ & $\hat{\lambda}$ & $\hat{\mathrm{k}}$ & $\hat{\mathrm{t}}_{\varphi}$ \\
\hline 1 & $\begin{array}{l}06 \\
09 \\
12 \\
15 \\
18\end{array}$ & $\begin{array}{l}47,7 \\
45,9 \\
46,1 \\
42 \\
35\end{array}$ & $\begin{array}{l}0,518 \\
0,540 \\
0,711 \\
0,706 \\
0,671\end{array}$ & $\begin{array}{r}21.10 \\
0.30 \\
0.37 \\
1.00 \\
4.40\end{array}$ \\
\hline 2 & $\begin{array}{l}06 \\
09 \\
12 \\
15 \\
18\end{array}$ & $\begin{array}{c}40,1 \\
37,7 \\
33,9 \\
29,3 \\
*\end{array}$ & $\begin{array}{c}0,468 \\
0,416 \\
0,544 \\
0,651 \\
*\end{array}$ & $\begin{array}{c}23.16 \\
23.20 \\
24.58 \\
24.28 \\
*\end{array}$ \\
\hline 3 & $\begin{array}{l}06 \\
09 \\
12 \\
15 \\
18\end{array}$ & $\begin{array}{c}38,5 \\
35,7 \\
37 \\
34 \\
*\end{array}$ & $\begin{array}{c}0,708 \\
0,502 \\
0,529 \\
0,638 \\
*\end{array}$ & $\begin{array}{c}22.09 \\
23.56 \\
1.44 \\
1.33 \\
*\end{array}$ \\
\hline 4 & $\begin{array}{l}06 \\
09 \\
12 \\
15 \\
18\end{array}$ & $\begin{array}{l}36,1 \\
34,7 \\
44,7 \\
44,9 \\
33,1\end{array}$ & $\begin{array}{l}0,408 \\
0,680 \\
0,698 \\
0,658 \\
0,724\end{array}$ & $\begin{array}{r}19.20 \\
23.56 \\
0.36 \\
0.46 \\
1.46\end{array}$ \\
\hline 5 & $\begin{array}{l}06 \\
09 \\
12 \\
15 \\
18\end{array}$ & $\begin{array}{l}37,7 \\
40,8 \\
46 \\
63,2 \\
36\end{array}$ & $\begin{array}{l}0,546 \\
0,386 \\
0,766 \\
0,883 \\
1,085\end{array}$ & $\begin{array}{r}22.41 \\
0.47 \\
0.43 \\
1.42 \\
1.43\end{array}$ \\
\hline 6 & $\begin{array}{l}06 \\
09 \\
12 \\
15 \\
18\end{array}$ & $\begin{array}{l}42,1 \\
37,8 \\
41,1 \\
45 \\
18,7\end{array}$ & $\begin{array}{l}0,439 \\
0,636 \\
0,870 \\
0,998 \\
0,931\end{array}$ & $\begin{array}{r}19.51 \\
1.05 \\
1.22 \\
1.45 \\
2.17\end{array}$ \\
\hline 7 & $\begin{array}{l}06 \\
09 \\
12 \\
15 \\
18\end{array}$ & $\begin{array}{l}41,4 \\
32,7 \\
30,7 \\
31,6 \\
24,6\end{array}$ & $\begin{array}{l}0,185 \\
0,511 \\
0,788 \\
0,690 \\
0,689\end{array}$ & $\begin{array}{r}21.18 \\
0.44 \\
1.17 \\
1.19 \\
1.59\end{array}$ \\
\hline 8 & $\begin{array}{l}06 \\
09 \\
12 \\
15 \\
18\end{array}$ & $\begin{array}{l}33,1 \\
51,2 \\
50,3 \\
62,3 \\
56,7\end{array}$ & $\begin{array}{l}0,292 \\
0,312 \\
0,609 \\
0,680 \\
0,713\end{array}$ & $\begin{array}{r}22.05 \\
2.41 \\
2.46 \\
1.25 \\
1.33\end{array}$ \\
\hline 9 & $\begin{array}{l}06 \\
09 \\
12 \\
15 \\
18\end{array}$ & $\begin{array}{c}* \\
32,6 \\
36,9 \\
40,6 \\
32,1\end{array}$ & $\begin{array}{c}* \\
0,297 \\
0,434 \\
0,675 \\
0,672\end{array}$ & $\begin{array}{c}* \\
1.07 \\
1.17 \\
1.10 \\
1.52\end{array}$ \\
\hline
\end{tabular}

$\hat{\lambda}$ : nombre moyen de prises d'aliment par jour ; $\hat{k}$ : indice d'étalement de l'activité alimentaire ; $\hat{\mathrm{t}}_{\varphi}$ : moment du maximum d'activité alimentaire (h et $\left.\mathrm{min}\right) ;{ }^{*}$ : données manquantes. 


$$
\begin{gathered}
-\hat{\mathrm{k}}_{\mathrm{ij}}=\mu^{\prime}+\delta \mathrm{t}_{\mathrm{i}}+\beta_{\mathrm{j}}+\mathrm{e}_{\mathrm{ij}} \quad \mathrm{e}_{\mathrm{ij}} \sim \mathcal{N}\left(0, \sigma^{2}\right) \\
\text { avec } \hat{\mu}^{\prime}=0,2238 \\
\hat{\delta}=0,0326 \\
\hat{\sigma}=0,1198
\end{gathered}
$$

où $\hat{\mu}^{\prime}, \hat{\delta}$ et $\hat{\sigma}$ sont respectivement les estimateurs de $\mu^{\prime}$, $\delta$ et $\sigma$

- $\hat{\mathrm{t}}_{\phi}=\mu^{\prime}+\alpha_{\mathrm{i}}^{\prime}+\delta \mathrm{t}_{\mathrm{i}}+\gamma \mathrm{t}_{\mathrm{i}}^{2}+\mathbf{e}_{\mathrm{ij}} \quad \mathrm{e}_{\mathrm{ij}} \sim \mathcal{N}\left(0, \sigma^{2}\right)$

$$
\text { avec } \begin{aligned}
\hat{\mu}^{\prime} & =15,635 \\
\hat{\delta} & =1,271 \\
\hat{\gamma} & =-0,039 \\
\hat{\alpha}_{1}^{\prime} & =-0,38, \hat{\alpha}_{2}^{\prime}=0,77, \hat{\alpha}_{3}^{\prime}=-0,05, \alpha_{4}^{\prime}=-0,70, \alpha_{5}^{\prime}=0,36 \\
\hat{\sigma} & =0,932
\end{aligned}
$$

où $\hat{\mu}^{\prime}, \hat{\delta}, \hat{\gamma}, \hat{\alpha}_{i}^{\prime}$ et $\hat{\sigma}$ sont respectivement les estimateurs de $\mu^{\prime}, \delta$, $\gamma, \alpha_{i}^{\prime}$ et $\sigma$.

L'affinage du modèle n'est pas de même nature pour $k$ et $t_{\phi}$. Pour $k$, l'influence de l'âge peut être uniquement décrite par une fonction simple de $t_{i}$. Ce n'est pas le cas pour $t_{\phi}$ où une régression quadratique de $\alpha_{i}$ sur $t_{j}$ et $t_{i}^{2}$ est insuffisante pour décrire l'influence de l'âge. Il faut introduire $\alpha_{i}^{\prime}$ dans le modèle pour expliquer l'écart à la régression quadratique de $t_{\phi}$ sur l'âge pour le niveau i de ce facteur. Notons que dans ces deux modèles $\mu^{\prime}$ ne peut être interprété comme une "moyenne générale " puisque la moyenne est respectivement

$$
\mu^{\prime}+\delta\left(\sum_{i=1}^{5} t_{i} / 5\right) \text { et } \mu^{\prime}+\delta\left(\sum_{i=1}^{5} t_{i} / 5\right)+\gamma\left(\sum_{i=1}^{5} t_{i}^{2} / 5\right)
$$

pour $k$ et $t_{\phi}$.

Ces résultats quantifient les tendances observées sur le tableau 1 et mettent en évidence la concentration progressive de l'activité alimentaire du lapin en fonction de l'âge. Cette concentration est accompagnée d'un déplacement de l'activité du début vers le milieu de la phase d'obscurité. Ainsi à 6 semaines, le lapin type a une activité alimentaire répartie autour de $21 \mathrm{~h} 30$ dont la dispersion est mesurée par un coefficient $k$ de 0,419 , alors qu'à 18 semaines, cette activité est répartie autour de 2 h 15 avec un coefficient $k$ de 0,810. Par contre, l'âge ne semble pas avoir d'influence sur le nombre de prises d'aliment par $24 \mathrm{~h}$. Cependant, pour ce dernier paramètre, l'importance de la variabilité inter-lapins masque peut-être la variabilité due à l'âge. La figure 4 résume bien l'ensemble des résultats obtenus à l'aide du processus de Poisson périodique et montre l'évolution moyenne de $h(t)$ en fonction de l'âge.

\section{Discussion. Conclusions.}

Les estimations non paramétriques de la densité mettent en évidence l'évolution de la répartition nycthémérale des prises d'aliment avec l'âge. Une répartition bimodale existe chez la plupart des animaux à 6 semaines et chez tous à 9 semaines puis tend à s'estomper vers la quinzième semaine en même temps qu'on observe une concentration progressive de l'activité alimentaire accompagnée d'un déplacement du maximum d'activité vers le milieu de la phase obs- 




FIG. 4. - Evolution moyenne en fonction de l'âge de la densité $h(t)$ calculée à partir du modèle de processus de Poisson périodique.

$6^{\mathrm{e}}$ semaine $: \ldots ; 9^{\mathrm{e}}$ semaine : ....................; $12^{\mathrm{e}}$ semaine :

$15^{\mathrm{e}}$ semaine : $\ldots .+\cdot \ldots+. ; 18^{\mathrm{e}}$ semaine $: \ldots \ldots$. $\ldots$ La zone ombrée correspond à la période d'obscurite.

cure. Cette concentration de l'activité, ainsi que le déplacement du maximum d'activité sont bien décrits par les variations des paramètres calculés à l'aide du modèle de processus de Poisson périodique.

Les résultats obtenus tendent à confirmer l'hypothèse de l'existence d'une phase de "maturation » du comportement alimentaire après le sevrage avec acquisition progressive des caractéristiques observées chez l'adulte (Reyne et al., 1980 ) et confirment l'accentuation avec l'âge de l'aspect nocturne des consommations décrit par Prud'hon et al. (1975a).

Le pic d'activité qui précède l'extinction des lumières chez l'animal jeune pourrait s'expliquer par le fait qu'à cet âge, la vitesse de croissance et les besoins sont importants. En effet, différentes publications tendent à montrer que les modifications des quantités ingérées dans différentes situations expérimentales portent surtout sur la période qui précède ou suit immédiatement l'extinction de la lumière (Prud'hon, 1976; Geiselman et al., 1980). La présence chez un animal à 6 semaines d'une deuxième période très marquée de moindre activit $€$ zu cours de la phase obscure suggère chez cet animal une deuxième période de caecotrophie dont Jilge (1979a et b) a montré l'existence chez 20 à 30 p. 100 des animaux adultes étudiés.

La forme de la densité $h(t)$ donnée par le modèle de processus de Poisson périodique (fig. 4) n'est sans doute pas très bien adaptée à la répartition des prises d'aliment chez le jeune lapin avant la quinzième semaine. En effet, elle possède un seul pic alors que les estimations non paramétriques (fig. 3 ) montrent 
que l'activité alimentaire avant cet âge présente souvent deux pics. Par ailleurs, le maximum et le minimum de la fonction $h(t)$ sont distants d'une demie-période, soit $12 \mathrm{~h}$ alors que les estimations non paramétriques montrent encore que cela n'est pas réalisé en général.

Cependant, ce modèle présente l'avantage d'avoir des paramètres interprétables et permet d'obtenir une information quantitative sur l'activité alimentaire qui facilite l'exploitation de ce type de données.

La répartition des instants de prise à partir de la quinzième semaine est assez bien décrite par le modèle utilisé. Ceci indiquerait que chez le Lapin, la probabilité d'apparition d'une prise d'aliment à un instant donné est peu modifiée par la présence de prises dans les instants précédents, la probabilité d'occurence d'une prise étant essentiellement déterminée par le rythme nycthéméral. Toutefois l'existence chez le Lapin d'une corrélation significative mais faible entre les quantités ingérées et les intervalles qui suivent (Sanderson et Vanderweele, 1975) montre qu'il serait intéressant d'introduire les quantités dans le modèle afin d'améliorer l'approche du phénomène. Ce travail devrait faire l'objet d'un article ultérieur.

Reçu en février 1982.

Accepté en août 1982.

\section{ANNEXE : QUELQUES DÉFINITIONS}

1) Densité d'un processus de Poisson. - Un processus de Poisson, qui est par définition un processus ponctuel, admet une densité $h(t)$ si le nombre moyen de points tombant dans l'intervalle $[\mathbf{a}, \mathbf{b}]$ quelconque a pour espérance $\int_{a}^{b} h(t) d t, h(t)$ étant une fonction positive. Le nombre de points du processus sera en moyenne plus grand quand la densité est plus élevée. Ainsi, dans notre cas, le maximum de $\mathrm{h}(\mathrm{t})$ correspondra à l'instant où la fréquence des prises d'aliment est la plus élevée.

2) Périodogramme des instants. - Le périodogramme est une fonction qui à la pulsation $\omega=2 \pi \lambda$ ( $\lambda$ est une fréquence) fait correspondre la quantité

$$
\mathrm{I}_{\mathrm{T}}(\omega)=\left(\sum_{\mathrm{i}=1}^{\mathrm{n}} \cos \omega \mathrm{t}_{\mathrm{i}}\right)^{2}+\left(\sum_{\mathrm{i}=1}^{\mathrm{n}} \sin \omega \mathrm{t}_{\mathrm{i}}\right)^{2} .
$$

En pratique, on calcule cette quantité pour les valeurs de $\lambda$ de la forme $\lambda=\frac{k}{T}$, où $k=1,2, \ldots m$ et $\mathrm{T}$ est la longueur du temps d'observation.

Dans notre cas, les suites $d$ 'instants de prise $t_{1}, t_{2}, \ldots, t_{n}$ des lapins durant une semaine sont considérées comme les réalisations d'un processus ponctuel.

$\mathrm{Si}$ nous supposons que le processus ponctuel est périodique et si $T$, la longueur du temps d'observation, est un multiple de la période $P$ du phénomène, alors $\mathrm{I}_{\mathrm{T}}\left(\frac{2 \pi k}{T}\right)$ est maximum pour $k=\frac{T}{P}$. Le périodogramme est donc un outil pour détecter les périodicités d'un processus ponctuel.

3) Estimation de densité. - Soit $x_{1}, x_{2}, \ldots, x_{n}$ un échantillon d'une variable aléatoire $X$ de densité de probabilité $f(x)$. Un problème naturel est celui de l'estimation de la fonction $f$ à partir de $x_{1}, x_{2}, \ldots$, $x_{n}$. Si nous supposons que $f$ dépend d'un ou plusieurs paramètres $\theta$, le problème se réduit à l'estimation de $\theta$. Si les hypothèses sur $f$ sont plus larges, par exemple, $f$ continue bornée, deux fois dérivable pour assurer une forme assez régulière à la fonction, alors la seule possibilité est de calculer un estimateur $\hat{f}_{n}(u)$ de la valeur $f(u)$ de la densité au point $u, u$ appartenant à D. D est l'ensemble des 
valeurs que peut éventuellement prendre la variable aléatoire $X$. En calculant $f_{n}(u)$ pour un nombre suffisamment important de points de $D$, il est possible d'obtenir des indications sur la forme de la fonction de densité $f$ et par conséquent sur la loi de répartition de la variable aléatoire $X$ sur $D$.

L'histogramme est un cas particulier d'estimateur non paramétrique de densité. Celui qui est utilisé dans ce travail à partir d'un $n$ échantillon $x_{1}, x_{2}, \ldots, x_{n}$ appartient à la classe des estimateurs dits "à noyau ". II est défini par l'équation :

$$
\hat{f}_{n}(u)=\frac{1}{n b_{n}} \sum_{i=1}^{n} g\left(\frac{x_{i}-u}{b_{n}}\right)
$$

où g est une fonction que nous appellerons noyau et qui a les propriétés suivantes :

- continue bornée

$-\int_{-\infty}^{+\infty} g(x) d x=1 \quad$ (c'est une densité de probabilité)

$-g(x)=g(-x)$ (c'est une fonction paire)

où $b_{1}, b_{2}, \ldots, b_{n}, \ldots$ est une suite de nombres telle que $b_{n}$ tend vers 0 quand $n$ tend vers l'infini.

Puisque $b_{n}$ tend vers 0 avec $n$, et que $g$ est intégrable, donc tend vers 0 à l'infini, $g\left(\frac{x_{i}-u}{b_{n}}\right)$ prend des valeurs importantes pour $u$ proche de $x_{i}$ et s'annule très vite quand $u$ s'éloigne de $x_{i}$, et ce d'autant plus vite que l'échantillon observé est plus grand. Le problème essentiel consiste à choisir $\mathrm{g}$ $d^{\prime}$ 'une part, la suite $b_{n} d$ 'autre part, afin d'obtenir simultanément un estimateur de biais et de variance minimum (Rosenblatt, 1971). Ceci se transpose sans grandes modifications à l'estimation de la densité d'un processus de Poisson.

\section{Références}

DE CASTRO J. M., 1975. Meal pettern correlations : facts and artifacts. Physiol. Behav., 15, 13-17.

DE CASTRO J. M., 1978. An analysis of the variance in meal patterning. Neurosci. Biobehav. Rev., 2, 301-310.

GEISELMAN P. J., MARTIN J. R., VANDERWEELE D. A., NOVIN D., 1980. Multivariate analysis of meal patterning in intact and vagotomized rabbits. J. comp. Physiol. Psychol., 94, 388-399.

HÖRNICKE H., DIETZEL F., AIGNER E., SCHULTE R., 1976. Alimentary behaviour in rabbits under different influences. Proc. 1 ${ }^{\text {st }}$ World's Rabbit Congr., Contrib. no. 13. Dijon.

HORTON B. J., TURLEY S. D., WEST C. E., 1974. Diurnal variation in the feeding pattern of rabbits. Life Sci., 15, 1895-1907.

JILGE B., 1979a. The entrainment of caecotrophy rhythm of the rabbit following 6 and $12 \mathrm{~h}$ phase-shifts of the zeitgeber. Chronobiologia, 6, 33-38.

JILGE B., 1979b. Zur circadianen Caecotrophie des Kaninchens. Z. Versuchstierkd., 21, 302-312.

JOLIVET E., 1980. Analyse de séries d'événements, 200-211. In JOLIVET E., LEGAY J. M., TOMASSONE R., Biométrie et temps, edited by INRA, Versailles.

LE MAGNEN J., DEVOS M., 1980. Parameters of the meal pattern in rats : their assessment and physiological significance. Neurosci. Biobehav. Rev., 4, suppl. 1, 1-11.

LE MAGNEN J., TALLON S., 1966. La périodicité spontanée de la prise d'aliments ad libitum du rat blanc. J. Physiol., 58, 323-349.

PANKSEPP J., 1978. Analysis of feeding patterns : data reduction and theoretical implications, 143-166. In BOOTH D. A., Hunger models, by Acad. Press, London.

PRUD'HON M., 1976. Comportement alimentaire du lapin soumis aux températures de 10, 20 et $30^{\circ} \mathrm{C}$. Proc. 1st World's Rabbit Congr., Contrib. no. 14. Dijon.

PRUD'HON M., CARLES Y., GOUSSOPOULOS J., KOEHL P. F., 1972. Enregistrement graphique des consommations d'aliments solides et liquides du lapin domestique nourri ad libitum. Ann. Zootech., 21, 451-460. 
PRUD'HON M., CHERUBIN M., GOUSSOPOULOS J., CARLES Y., 1975a. Evolution au cours de la croissance des caractéristiques de la consommation d'aliments solides et liquides du lapin domestique nourri ad libitum. Ann. Zootech., 24, 289-298.

PRUD'HON M., CHERUBIN M., CARLES Y., GOUSSOPOULOS J., 1975b. Effets de différents niveaux de restriction hydrique sur l'ingestion d'aliments solides par le lapin. Ann. Zootech., 24, 299-310.

REYNE Y., CARLES Y., GOUSSOPOULOS J., 1980. Comportement alimentaire du lapin croisé Néozélandais $\times$ Garenne âgé de 12 semaines. Proc. $2^{\text {nd }}$ World's Rabbit Congr., Barcelona, avril 1980, vol. 2, 111-118.

ROSENBLATT M., 1971. Curve estimates. Ann. math. Stat., 42, 1815-1842.

SANDERSON J. D., VANDERWEELE D. A., 1975. Analysis of feeding patterns in normal and vagotomized rabbits. Physiol. Behav., 15, 357-364.

THOMAS D. W., MAYER J., 1968. Meal taking and regulation of food intake by normal and hypothalamic hyperphagic rats. J. comp. Physiol. Psychol., 66, 642-653. 